\title{
Impact of COVID-19 Pandemic on Cardiac Electronic Device Management and Role of Remote Monitoring
}

Michele Magnocavallo, MDª,b, Giampaolo Vetta, MDa, Alessia Bernardini, MDa ${ }^{a}$, Agostino Piro, MDa ${ }^{a}$, Maria Chiara Mei, MDa, Martina Di lorio, MDa ${ }^{a}$ Marco Valerio Mariani, MDa, Domenico G. Della Rocca, MD, PhD ${ }^{b}$, Paolo Severino, MD, PhDa , Raffaele Quaglione, $\mathrm{MD}^{\mathrm{a}}$, Giuseppe Giunta, $\mathrm{MD}^{\mathrm{a}}$, Cristina Chimenti, MD, PhD ${ }^{a}$, Fabio Miraldi, MD ${ }^{a}$, Carmine Dario Vizza, MD ${ }^{a}$, Francesco Fedele, MD ${ }^{a}$, Carlo Lavalle, MD ${ }^{a, *}$

\section{KEYWORDS}

- COVID-19 • Remote monitoring • Telemedicine • CIED • Telehealth • Pacemaker

- Implantable cardiac defibrillator

\section{KEY POINTS}

- The aim of remote monitoring is to optimize the clinical management of CIED patients, improve quality of life, and reduce hospitalization and emergency department access.

- A well-established organizational model should include an adequately structured team, a valid integration with the primary health care centers, and an appropriate response to clinical alerts.

- The development and refinement of telemedicine during the pandemic period suggest that remote monitoring should be recommended for all CIED patients.

\section{INTRODUCTION}

On December 31, 2019, a cluster of pneumonia cases of unknown origin was reported in the city of Wuhan; severe acute respiratory syndrome coronavirus 2 (SARS-CoV-2) was then discovered as the causative agent of the respiratory disease named coronavirus disease 2019 (COVID-19). ${ }^{1}$ The epidemic spread rapidly through China and subsequently to the rest of the world, leading the World Health Organization to declare the pandemic state on March 11, 2020.

COVID-19 has caused a global impact on public health services that led to the reorganization of hospital settings, including in-office visits for patients with cardiac implantable electronic device
(CIED). Remote monitoring (RM) of CIED patients represents an appropriate strategy to minimize any potential risk of virus exposure for patients and health care providers, without compromising the quality of care. ${ }^{2-5}$ RM offers access to the same information as an in-office visit and may contribute to the early detection of atrial and ventricular arrhythmias, ${ }^{6,7}$ prevent heart failure (HF) decompensation, and manage device-related issues. ${ }^{8-12} \mathrm{RM}$ has also confirmed its usefulness in decreasing the hospitalization rate and improving clinical outcomes. ${ }^{13}$ Moreover, since the onset of the COVID-19 pandemic, physicians have suspended nonurgent scheduled visits and made a rapid transition to virtual visits (VV). ${ }^{14-16}$ Thanks

\footnotetext{
a Department of Cardiovascular/Respiratory Diseases, Nephrology, Anesthesiology, and Geriatric Sciences, Policlinico Umberto I, Sapienza University of Rome, Rome, Italy; ${ }^{b}$ Texas Cardiac Arrhythmia Institute, St. David's Medical Center, 3000 North IH-35, Suite 720, Austin, TX 78705, USA

* Corresponding author.

E-mail address: carlolavalle@uniroma1.it
} 
to the technological improvement, patients utilizing wearable sensors for the measurement of hemodynamic parameters (blood pressure and saturation, heart rate) and adopting virtual health platforms may be monitored directly from home without any risk of infection.

In this review, we provide an overview of the many possible applications of RM, its limitations and challenges in patients with CIED during the COVID-19 pandemic.

\section{THE ROLE OF RM IN CIED MANAGEMENT DURING COVID-19}

RM of CIED patients has become increasingly popular in clinical practice, especially during the COVID-19 pandemic. ${ }^{17}$ Indeed, in the new guidelines of the European Society of Cardiology (ESC) on cardiac pacing, RM is recommended to reduce the number of in-office follow-up in patients with pacemaker (PMK) who have difficulties to attend in-person visits. RM may also be useful in case of a device component that has been recalled or is on advisory, to enable early detection of actionable events in patients at high risk. ${ }^{18} \mathrm{RM}$ provides the same information as an in-person visit ensuring an early identification of cardiac arrhythmias, such as ventricular tachycardias or atrial fibrillation, device therapy, and devicerelated issues like lead malfunction and early battery discharge. ${ }^{19-22}$ Additional benefits were also demonstrated among HF patients in terms of preventing unfavorable cardiovascular events and reducing hospital readmissions. ${ }^{12,23}$

Although guidelines recommended the use of RM for the follow-up of patients with CIED, the coverage of RM was limited because of the organizational problems of health care systems and reimbursement issues. ${ }^{18,20}$ The COVID-19 pandemic forced all health care providers to minimize interpersonal contacts to limit the spread of the virus, which led to a total reshaping of outpatient cardiology management and accelerated the deployment and widespread use of RM. ${ }^{24}$ Indeed, the consensus document of the Heart Rhythm Society (HRS) and ESC for the management of cardiovascular disease during the COVID-19 pandemic recommended that RM should replace in-office visits for device interrogation and, whenever possible, postpone the scheduled in-person visit. ${ }^{3,4,14,24,25}$

A questionnaire-based survey by the European Heart Rhythm Association (EHRA) to assess the influence of the COVID-19 pandemic on RM in CIEDs demonstrated a strong implementation of RM in patients with $\mathrm{PMKs}$ and implantable loop recorders (ILRs; PMK 24.2 vs 39.9\%; $P=.002$; ILR
61.5 vs $73.5 \% ; P=.028$ ). A nonsignificant increasing trend was registered for RM of cardiac resynchronization therapy-pacemaker (CRT-P) devices (44.5 vs $55 \%$; $P=.063$ ), implantable cardioverter defibrillators (ICDs; 65.2 vs $69.6 \%$; $P=.408$ ) and CRT-defibrillators (CRT-D; 65.2 vs $68.8 \% ; P=.513) .{ }^{26}$

Home delivery of the transmitter for RM should be preferred over in-office delivery, as it limits the exposure of patients to the hospital environment. To date, home delivery of transmitter is feasible for Boston Scientific and Abbott PM and ICDs, as well as for the latest Medtronic CIEDs with BlueSync. ${ }^{24}$ As demonstrated in a recent multicenter study, the communicator LATITUDE was home delivered to 1324 patients from 49 different Italian centers and successful activation through telephone training was achieved in 92\% of cases. ${ }^{27}$ Moreover, De Larochellière and colleagues confirmed that switching from a followup model with in-person visits to an RM model did not impair the management of ICD patients, and significantly reduced the number of inperson visits. ${ }^{28}$ In Fig. 1, we summarized a protocol for setting up and managing RM during the COVID-19 pandemic. When the device is suitable for RM, home delivery of the transmitter is an appropriate strategy for minimizing any potential risk of virus exposure for patients and health care providers.

Overall, the available evidence confirmed that RM is an easy-to-use and effective tool for the management of CIED patients even during the pandemic and demonstrated that the home delivery and activation of communicators without an inpatient visit is a potential opportunity to further extend RM in the future.

\section{SUPPLEMENTATION OF TELECONSULTATION IN CIED PATIENTS}

The ESC and HRS consensus documents stated that in-person visits should be replaced by telemedicine consultations in order to prevent the spread of the virus among cardiovascular patients. ${ }^{3,24}$ Indeed, the worldwide survey by Han and colleagues about the use of eHealth technologies during the COVID-19 pandemic showed a significant increase in the use of teleconsultations in the management of cardiological patients $(5.9 \%$ vs $58.6 \% ; P<.001)$ for all types of consultations compared with the prepandemic period. ${ }^{25}$

Supplementing teleconsultation in CIED patients could be a key tool in the management of these patients during the COVID-19 pandemic, especially for those affected by HF. ICD and CRT have the capability of monitoring HF by measuring 


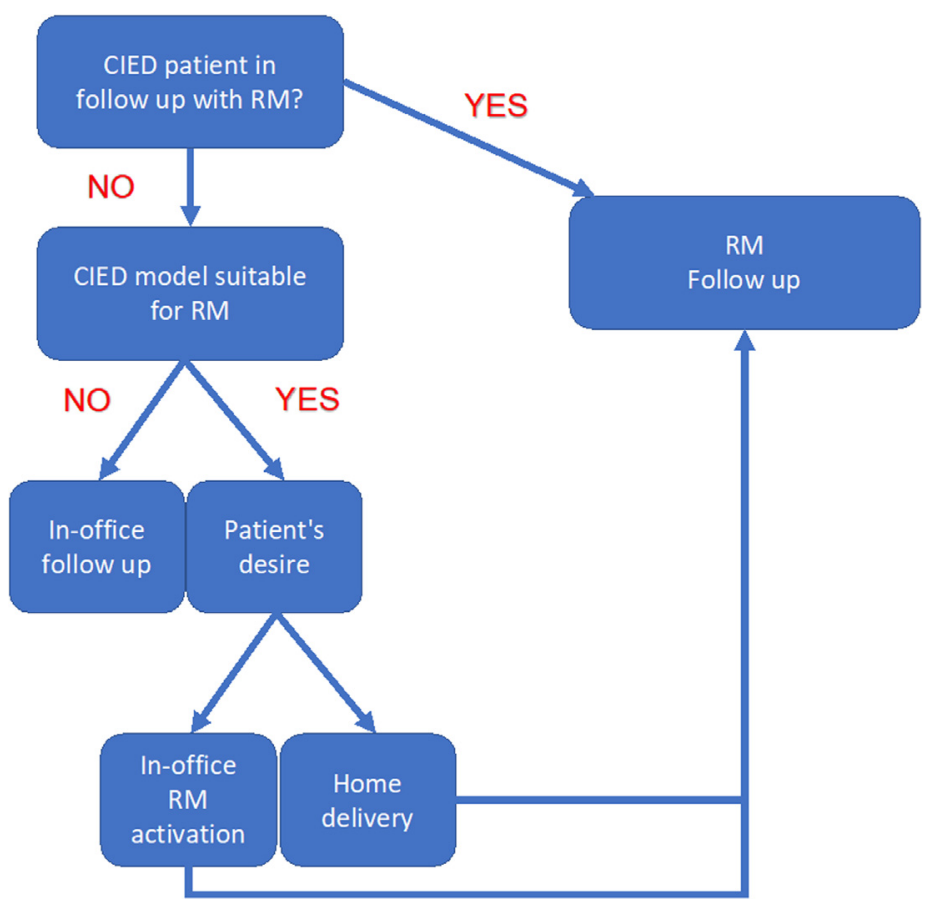

Fig. 1. Remote monitoring setup protocol. CIED, cardiac implantable electronic device; RM, remote monitoring.

thoracic impedance (Optivol, Medtronic; CorVue, Abbott) ${ }^{29}$ or by integration of several indices (HeartLogic, Boston Scientific). ${ }^{30}$ Nevertheless, guidance statements issued by experts in electrophysiology and HF recommend that every effort should be made to convert in-office visits to telehealth and VV. Specifically, the VVs used for decades to reach remote communities, ${ }^{31}$ but less commonly used in advanced health care systems, have now emerged as the cornerstone of ambulatory care in all subspecialties. ${ }^{32}$ The potential benefits of VV for HF patients are providing access to care and medical advice, which would be otherwise difficult to obtain and reducing in-person exposure to SARS-CoV-2. Cardiac rhythm professionals are advantaged by having wireless technology available to transmit monitored information to keep them connected. ${ }^{33}$ Moreover, V Vs have the advantage of detecting and alerting caregivers about relevant parameter changes, allowing earlier hospitalization of the patient, even in a presymptomatic phase. ${ }^{34} \mathrm{~A}$ flowchart for $\mathrm{V}$ is summarized in Fig. 2; VV was recommended in patients with atrial arrhythmias, alert for HF decompensation and nonsustained ventricular tachycardia.

Overall, new technologies and digital platforms to aid in remote care should be developed and further research on the role of telehealth, continuous data collecting, advanced automotive features, and RM is needed to guide best practices.

\section{PATIENT ACCEPTABILITY AND SATISFACTION OF RM DURING COVID-19}

As in any health care interaction, patient involvement plays an important role, and in the case of $\mathrm{RM}$, active participation is fundamental. Patients must adhere to transmission timetables and keep in contact with the physician to guarantee a successful health care system based on RM. Therefore, from a positive reciprocal interaction between patient and caregiver usually derives a high acceptability and satisfaction. From the point of view of the patient, especially during the COVID19 period, RM should be ease of use, ${ }^{35-37}$ even when manual transmission of the data is requested, and guarantee a positive relationship with their health care provider at enrollment and during all the monitored period. ${ }^{36,38-40}$ The Home Monitoring Acceptance and Satisfaction Questionnaire is administered to evaluate the acceptability and satisfaction of RM (HoMASQ) and showed that ICD patients had a higher level of acceptance and satisfaction than patients with PMK. ${ }^{35,39}$ Moreover, RM was demonstrated to be easy to use and well accepted even for older people and patients with a low level of scholarity. ${ }^{41}$ Otherwise, the most frequent causes of noncompliance seem to be:

- age-related: age under 40 years was associated with lower compliance. 


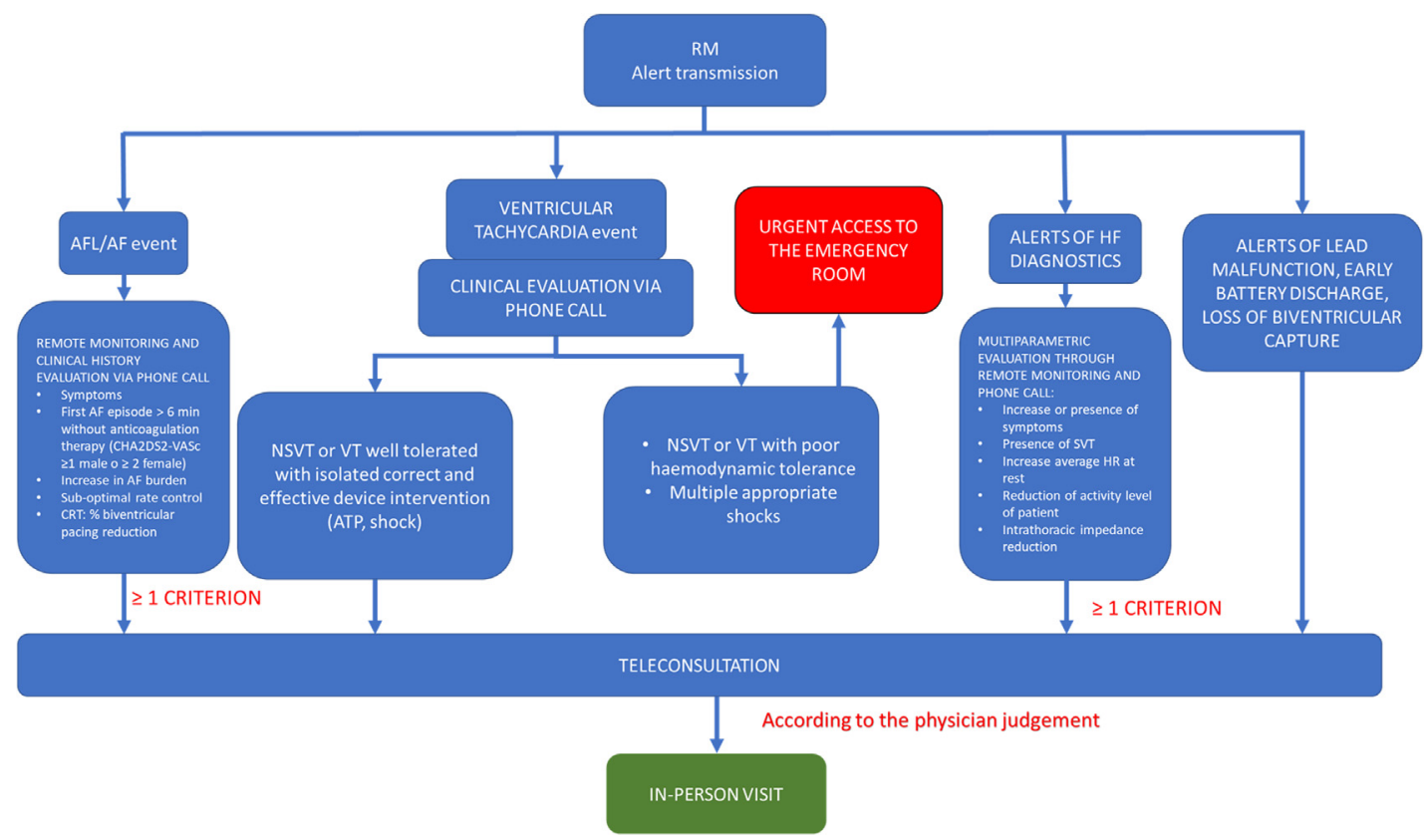

Fig. 2. Remote monitoring management protocol. $A F$, atrial fibrillation; $A F L$, atrial flutter; $H F$, heart failure; $H R$, heart rate; NSVT, non-sustained ventricular tachycardia; RM, remote monitoring; SVT, supraventricular tachycardia; VT, ventricular tachycardia.

- Health care systems-related: high volume clinics were associated with better compliance.

- Device-related: wireless devices are characterized by a better compliance compared with those requiring use of a wand. ${ }^{42}$

The institution of an RM patient agreement supported by the HRS enhances the compliance because patients can freely share information and experiences. ${ }^{17}$

During the COVID-19 pandemic, also the delivery of the communicator for RM could reduce the risk of contagion and influence patient's acceptability. Piro and colleagues demonstrated that home delivery of the communicator and intensive transtelephonic support for its activation resulted in an easy understanding of the device activation process, as well as high satisfaction with the use of the transmitter. ${ }^{2}$ In addition, despite the ongoing pandemic and national lockdown, patients referred a sense of security and expressed interest in continuing with RM; also, in-office modem delivery and activation was associated with a higher prevalence of anxiety symptoms due to COVID-19 pandemic, compared with home modem delivery. ${ }^{2,27}$

In conclusion, several studies showed a high level of patient satisfaction and compliance, making it possible to extend this form of management to a growing volume of patients, especially in times of pandemics.

\section{RM FOR CIED PATIENTS WITH OTHER COMORBIDITIES}

CIED patients are usually affected by multiple comorbidities: neurologic syndromes, chronic kidney disease, chronic obstructive pulmonary disease, diabetes mellitus, and other endocrinological disorders. New technologies and the adaptation of existing telemedicine tools represent an alternative option for an integrated monitoring. ${ }^{3,32}$ For example, diabetes patients need recurrent medical consultations to optimize drug therapy and blood sugar levels, and telemedicine can be a valuable alternative, especially during a pandemic when contacts need to be limited. ${ }^{43}$ To confirm the effectiveness of RM for diabetes management, a recent meta-analysis demonstrated a reduction in glycated hemoglobin in the RM group compared with controls. ${ }^{44}$ Moreover, continuous glucose monitoring is effective in the management of high-risk patients with type 1 diabetes mellitus without any diabetic ketoacidosis. ${ }^{45}$

Telemedicine also spread into the field of neurology and a telestroke unit was established to allow remote assessment of patients with suspected stroke to minimize unnecessary in-person 
visits. ${ }^{46}$ The latest evidence demonstrated that in-hospital management of end-stage renal disease patients increased the risk of infection up to 4 times compared with telemedicine-based home management and was more expensive. Similarly, RM appeared effective in the rehabilitation and management of chronic obstructive pulmonary disease patients, leading to a reduction in hospitalizations and emergency department visits. ${ }^{47}$

All this evidence shows how the pandemic escalated the adoption of telemedicine and all aspects of digital health, and this new reality is now likely to define medicine in the future not only in cardiology but also in other branches of medicine.

\section{ECONOMIC ASPECTS}

In addition to primary analyses focusing on cardiovascular outcomes (hospitalizations, cardiovascular death, overall death), another important aspect to consider for the adoption of digital health solutions is their impact on health care expenditure. ${ }^{48,49}$ Owing to the outbreak of the SARSCoV-2, a prompt reorganization of health care services was necessary with a related new economicfinancial business plan.

The TARIFF study demonstrated that the overall mean annual cost per patient for in-office followup was significantly higher than an RM-based one $(-53.87 \%$ in the RM group). The main reason for cost reduction is due to the cost of cardiovascular hospitalizations $(€ 886.67 \pm € 1979.13$ vs $€ 432.34 \pm € 2488.10 ; P=.0030){ }^{50}$ The same findings were reported in the EVOLVO study, a multicenter clinical trial aiming at measuring the benefits of RM for HF patients with ICDs. The results of this study showed that RM was costeffective with an average saving of $€ 888.10$ per patient. ${ }^{48}$ Notably, cost-effectiveness between countries varied considerably depending on whether there was specific reimbursement for RM services. In fact, there was heterogeneity among countries, with RM generating less profits for providers in the absence of specific reimbursements and similar or increased profits in cases such reimbursements existed. ${ }^{51}$ Indeed, according to a recent European survey, the absence of reimbursement in many countries is generally considered the major barrier to the implementation of RM in standard practice. ${ }^{20}$

RM was cost-effective for health care systems because of lower follow-up costs and hospitalization reductions; the future challenge will be a more uniform deployment of appropriate reimbursement systems.

\section{SUMMARY}

The COVID-19 pandemic imposed challenges to the traditional rules of access and delivery of health care worldwide. ${ }^{52}$ It accelerated the adoption of telemedicine and digital health, confirming a new era in the management of CIED patients. Patient outcomes could be improved with device-based intensive monitoring compared with traditional in-clinic follow-up at regular intervals. ${ }^{53}$ The pandemic experience promoted the search for alternative solutions for an effective patient follow-up, such as validation of digital technologies, data management strategies, implementation of predictive analytics, cybersecurity, development of limited forms of remote CIED programming, and reimbursement. ${ }^{19,51,54}$

\section{CLINICS CARE POINTS}

- Remote Monitoring should be proposed in all CIED patients.

- Remote Monitoring is safe and effective also during COVID pandemic.

- Virtual Visit might be used in patients with multiple cardiac comorbidities.

\section{DISCLOSURES}

The authors have nothing to disclose.

\section{REFERENCES}

1. Zhu N, Zhang D, Wang W, et al. A novel Coronavirus from patients with pneumonia in China, 2019. N Engl J Med 2020;382:727-33.

2. Piro A, Magnocavallo M, Della Rocca DG, et al. Management of cardiac implantable electronic device follow-up in COVID-19 pandemic: lessons learned during Italian lockdown. J Cardiovasc Electrophysiol 2020;31:2814-23.

3. Varma N, Marrouche NF, Aguinaga L, et al. HRS/ EHRA/APHRS/LAHRS/ACC/AHA worldwide practice update for telehealth and arrhythmia monitoring during and after a pandemic. EP Europace 2021;23: 313.

4. Mohanty S, Lakkireddy D, Trivedi C, et al. Creating a safe workplace by universal testing of SARS-CoV-2 infection in asymptomatic patients and healthcare workers in the electrophysiology units: a multicenter experience. J Interv Card Electrophysiol 2020. https://doi.org/10.1007/s10840-020-00886-9.

5. Della Rocca DG, Magnocavallo M, Lavalle C, et al. Evidence of systemic endothelial injury and 
microthrombosis in hospitalized COVID-19 patients at different stages of the disease. J Thromb Thrombolysis 2020. https://doi.org/10.1007/s11239-02002330-1.

6. Della Rocca DG, Santini L, Forleo GB, et al. Novel perspectives on arrhythmia-induced cardiomyopathy: pathophysiology, clinical manifestations and an update on invasive management strategies. Cardiol Rev 2015;23:135-41.

7. Chen Q, Xu J, Gianni C, et al. Simple electrocardiographic criteria for rapid identification of wide grs complex tachycardia: the new limb lead algorithm. Heart Rhythm 2020;17:431-8.

8. Forleo GB, Panattoni G, Schirripa V, et al. Device monitoring of heart failure in cardiac resynchronization therapy device recipients: a single-center experience with a novel multivector impedance monitoring system. J Cardiovasc Med 2013;14: 726-32.

9. Ong MK, Romano PS, Edgington S, et al. Effectiveness of remote patient monitoring after discharge of hospitalized patients with heart failure: the better effectiveness after transition-heart failure (BEATHF) randomized clinical trial. JAMA Intern Med 2016;176:310.

10. García-Fernández FJ, Osca Asensi J, Romero R, et al. Safety and efficiency of a common and simplified protocol for pacemaker and defibrillator surveillance based on remote monitoring only: a long-term randomized trial (RM-ALONE). Eur Heart J 2019;40: 1837-46.

11. Pignalberi C, Mariani MV, Castro A, et al. Sporadic high pacing and Shock impedance on remote monitoring in Hybrid implantable cardioverterDefibrillator systems: clinical impact and management. Heart Rhythm 2021;18:1292-300.

12. Boehmer JP, Hariharan R, Devecchi FG, et al. A multisensor algorithm predicts heart failure events in patients with implanted devices. JACC: Heart Fail 2017:5:216-25.

13. Hindricks G, Taborsky M, Glikson M, et al. Implantbased multiparameter telemonitoring of patients with heart failure (IN-TIME): a Randomised Controlled trial. Lancet 2014;384:583-90.

14. AIAC Ricerca Network Investigators, Boriani G, Palmisano P, Guerra F, et al. Impact of COVID-19 pandemic on the clinical activities related to arrhythmias and electrophysiology in Italy: results of a survey promoted by AIAC (Italian association of arrhythmology and cardiac pacing). Intern Emerg Med 2020;15:1445-56.

15. Hollander JE, Carr BG. Virtually perfect? Telemedicine for covid-19. N Engl J Med 2020;382:1679-81.

16. Lakkireddy DR, Chung MK, Gopinathannair R, et al. Guidance for cardiac electrophysiology during the COVID-19 pandemic from the heart rhythm Society COVID-19 Task Force; electrophysiology section of the American College of cardiology; and the Electrocardiography and arrhythmias Committee of the Council on clinical cardiology, American heart association. Heart Rhythm 2020;17:e233-41.

17. Slotwiner D, Varma N, Akar JG, et al. HRS expert consensus statement on remote interrogation and monitoring for cardiovascular implantable electronic devices. Heart Rhythm 2015;12:e69-100.

18. Glikson M, Nielsen JC, Kronborg MB, et al. ESC guidelines on cardiac pacing and cardiac resynchronization therapy. Eur Heart J 2021;2021. ehab364.

19. Saxon LA, Varma N, Epstein LM, et al. Factors influencing the decision to proceed to firmware upgrades to implanted pacemakers for cybersecurity risk mitigation. Circulation 2018;138:1274-6.

20. Mairesse GH, Braunschweig F, Klersy K, et al. Implementation and reimbursement of remote monitoring for cardiac implantable electronic devices in Europe: a survey from the health economics Committee of the European heart rhythm association. EP Europace 2015;17:814-8.

21. Della Rocca Domenico G, Albanese M, Placidi F, et al. Feasibility of automated detection of sleep apnea using implantable pacemakers and defibrillators: a comparison with simultaneous polysomnography recording. J Interv Card Electrophysiol 2019;56: 327-33.

22. Forleo GB, Tesauro M, Panattoni G, et al. Impact of continuous intracardiac st-segment monitoring on mid-term outcomes of ICD-Implanted patients with coronary artery disease. Early results of a prospective comparison with conventional ICD outcomes. Heart 2012;98:402-7.

23. Dang S, Dimmick S, Kelkar G. Evaluating the evidence Base for the Use of home telehealth remote monitoring in Elderly with heart failure. Telemed e-Health 2009; 15:783-96.

24. The European Society for Cardiology. ESC Guidance for the Diagnosis and Management of CV Disease during the COVID-19 Pandemic. Availlable at: https://www.escardio.org/Education/COVID-19and-Cardiology.

25. Han JK, Al-Khatib SM, Albert CM. Changes in the digital health landscape in cardiac electrophysiology: a pre-and peri-pandemic COVID-19 era survey. Cardiovasc Digital Health J 2021;2:55-62.

26. Simovic S, Providencia R, Barra S, et al. The use of remote monitoring of cardiac implantable devices during the COVID-19 pandemic: an EHRA physician survey. EP Europace 2021. https://doi.org/10.1093/ europace/euab215. euab215.

27. Magnocavallo M, Bernardini A, Mariani MV, et al. Home delivery of the communicator for remote monitoring of cardiac implantable devices: a multicenter experience during the Covid-19 lockdown. Pacing Clin Electrophysiol 2021;44:995-1003. 
28. De Larochellière H, Champagne J, Sarrazin J-F, et al. Findings of remote monitoring of implantable cardioverter defibrillators during the COVID-19 pandemic. Pacing Clin Electrophysiol 2020;43:1366-72.

29. Abraham WT, Compton S, Haas G, et al. Intrathoracic impedance $v$ s daily weight monitoring for predicting worsening heart failure events: results of the fluid accumulation status trial (FAST). Congest Heart Fail 2011;17:51-5.

30. Boehmer JP, Hariharan R, Devecchi FG, et al. A multisensor algorithm predicts heart failure events in patients with implanted devices: results from the MultiSENSE study. JACC Heart Fail 2017;5:216-25.

31. Bagchi S. Telemedicine in Rural India. Plos Med 2006;3:e82.

32. Sayed S. COVID-19 and diabetes; possible role of polymorphism and rise of telemedicine. Prim Care Diabetes 2021;15:4-9.

33. Steinberg JS, Varma N, Cygankiewicz I, et al. 2017 ISHNE-HRS expert consensus statement on ambulatory ECG and External cardiac monitoring/telemetry. Heart Rhythm 2017;14:e55-96.

34. Varma N, Epstein AE, Irimpen A, et al. TRUST investigators efficacy and safety of automatic remote monitoring for implantable cardioverter-defibrillator follow-up: the lumos-t safely reduces routine office device follow-up (TRUST) trial. Circulation 2010; 122:325-32.

35. Morichelli L, Porfili A, Quarta L, et al. Implantable cardioverter defibrillator remote monitoring is well Accepted and easy to use during long-Term followup. J Interv Card Electrophysiol 2014;41:203-9.

36. Ricci RP, Morichelli L, Quarta L, et al. Long-term patient Acceptance of and satisfaction with implanted device remote monitoring. Europace 2010;12:674-9.

37. Schoenfeld MH, Compton SJ, Mead $\mathrm{RH}$, et al. Remote monitoring of implantable cardioverter defibrillators: a prospective analysis. Pacing Clin Electrophysiol 2004;27:757-63.

38. Marzegalli M, Lunati M, Landolina M, et al. Remote monitoring of CRT-ICD: the multicenter Italian CareLink evaluation-ease of use, acceptance, and organizational implications. Pacing Clin Electrophysiol 2008;31:1259-64.

39. Petersen HH, Larsen MCJ, Nielsen OW, et al. Patient satisfaction and suggestions for improvement of remote ICD monitoring. J Interv Card Electrophysiol 2012;34:317-24.

40. Hindricks G, Elsner C, Piorkowski C, et al. Quarterly vs. Yearly clinical follow-up of remotely monitored recipients of prophylactic implantable cardioverterdefibrillators: results of the REFORM trial. Eur Heart J 2014;35:98-105.

41. Morichelli L, Ricci R, Sassi A, Quarta L, Porfili A, al CadedduNet. ICD remote monitoring is well Accepted and easy to use even for elderly. Eur Heart J 2011;32(Suppl. 1):32.
42. Rosenfeld LE, Patel AS, Ajmani VB, et al. Compliance with remote monitoring of ICDS/CRTDS in a real-world population. Pacing Clin Electrophysiol 2014;37:820-7.

43. Ghosh A, Gupta R, Misra A. Telemedicine for diabetes care in India during COVID19 pandemic and national lockdown period: guidelines for physicians. Diabetes Metab Syndr Clin Res Rev 2020;14:273-6.

44. Zhai Y-K, Zhu W-J, Cai Y-L, et al. Clinical- and costeffectiveness of telemedicine in type 2 diabetes mellitus: a systematic review and meta-analysis. Medicine (Baltimore) 2014;93:e312.

45. Peters AL, Garg SK. The silver lining to COVID-19: avoiding diabetic ketoacidosis admissions with telehealth. Diabetes Technology Ther 2020;22:449-53.

46. Majersik JJ, Reddy VK. Acute neurology during the COVID-19 pandemic: supporting the front line. Neurology 2020;94:1055-7.

47. Velayati F, Ayatollahi H, Hemmat M. A systematic review of the effectiveness of telerehabilitation interventions for therapeutic purposes in the elderly. Methods Inf Med 2020;59:104-9.

48. Zanaboni P, Landolina M, Marzegalli M, et al. Costutility analysis of the EVOLVO study on remote monitoring for heart failure patients with implantable defibrillators: randomized controlled trial. J Med Internet Res 2013;15:e106.

49. Guédon-Moreau L, Lacroix D, Sadoul N, et al. ECOST trial Investigators costs of remote monitoring vs. Ambulatory follow-Ups of implanted cardioverter defibrillators in the Randomized ECOST study. Europace 2014;16:1181-8.

50. Ricci RP, Vicentini A, D'Onofrio A, et al. Economic analysis of remote monitoring of cardiac implantable electronic devices: results of the health economics evaluation registry for remote follow-up (TARIFF) study. Heart Rhythm 2017;14:50-7.

51. Heidbuchel $H$, Hindricks $G$, Broadhurst $P$, et al. EuroEco (European health economic trial on home monitoring in ICD patients): a provider perspective in five european countries on costs and net financial impact of follow-up with or without remote monitoring. Eur Heart J 2015;36:158-69.

52. Udwadia ZF, Raju RS. How to protect the protectors: 10 lessons to learn for doctors fighting the COVID19 Coronavirus. Med J Armed Forces India 2020; 76:128-31.

53. Hindricks G, Varma N, Kacet S, et al. Daily remote monitoring of implantable cardioverter-defibrillators: Insights from the Pooled patient-level data from three Randomized Controlled trials (IN-TIME, ECOST, TRUST). Eur Heart J 2017;38:1749-55.

54. Slotwiner DJ, Abraham RL, Al-Khatib SM, et al. HRS white paper on interoperability of data from cardiac implantable electronic devices (CIEDs). Heart Rhythm 2019;16:e107-27. https://doi.org/10.1016/j. hrthm.2019.05.002. 